\title{
Research Paper \\ Effectiveness of Group Logotherapy on Death Anxiety and Life Expectancy of the Elderly Living in Boarding Houses in Kerman
}

\author{
Amir Hossein Hajiazizi ${ }^{1}$, Behnaz Bahmani², Nafiseh Mahdi ${ }^{3}{ }^{*}$ Vahid Manzari Tavakoli ${ }^{4}$, Adibeh Barshan ${ }^{5}$
}

1. Department of Counseling Psychology, School of Human Sciences, Assumption University, Bangkok, Thailand.

2. Department of Clinical Psychology, Faculty of Economics and Social Sciences, Bu-Ali Sina University, Hamedan, Iran.

3. Department of Educational Psychology, Faculty of Psychology \& Educational Sciences, Allameh Tabataba'i University, Tehran, Iran.

4. Department of Educational Sciences, Farhangian University of Kerman, Kerman, Iran.

5. Department of Psychology, Faculty of Psychology and Education, University of Tehran, Tehran, Iran.

Citation: Hajiazizi AH, Bahmani B, Mahdi N, Manzari Tavakoli V, Barshan A. [Effectiveness of Group Logotherapy on Death Anxiety and Life Expectancy of the Elderly Living in Boarding Houses in Kerman (Persian)]. Iranian Journal of Ageing. 2017; 12(2):220-231. http://dx.doi. org/10.21859/sija-1202220

: http://dx.doi.org/10.21859/sija-1202220

Received: 20 Jan. 2017

Accepted: 20 May 2017

Keywords:

Group logotherapy,

Death anxiety, Life

expectancy, Elderly

\section{A B STRACT}

Objectives Due to the growing elderly population, it is important to solve the problems faced by the elderly, including their mental health problems. Since the increase in death anxiety and the decrease in life expectancy is more common in the elderly, this study aimed to investigate the effectiveness of group logotherapy on death anxiety and life expectancy of the elderly living in boarding houses in Kerman.

Methods \& Materials The study population consisted of all elderly people in Kerman. By visiting two centers of Mehrazin and Amiralmo'menin in Kerman, 30 elderly people (18 female and 12 male) were selected and randomly assigned to experimental and control groups (each $n=15$ ). The experimental group was provided group logotherapy for ten sessions (each session of 90 minutes). The control group received no intervention. Both before and after the test, the participants were asked to respond to Miller's Life Expectancy Scale (1997) and Death Anxiety Scale. The data were analyzed using statistical methods such as frequency, percentage, mean and standard deviation, and covariance multivariate analysis.

Results The results showed that group therapy has a significant impact on death anxiety in the elderly $(\mathrm{P}<0.01)$. The group therapy was also found to affect the life expectancy of the elderly at a significance level of $\mathrm{P}<0.01$.

Conclusion Overall, based on the results of this research, group therapy was found to reduce death anxiety and life expectancy in elderly people living in boarding houses and subsequently, improve their mental health. Due to the special emphasis of logotherapy on the present and the meaning of suffering for the elderly, it is important to use it for promoting social well-being of older people.

\section{Extended Abstract}

\section{Objectives}

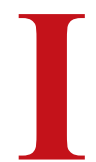

thas been estimated that by 2030 , the elderly population of the world will increase from $9 \%$ to $16 \%$ and from $5.6 \%$ to $17.5 \%$ in Iran

[1]. Thus, it is essential to pay attention to this age group while improving their quality of life. Given the growing population of the elderly people, it is necessary to resolve their problems including their psychological problems [2]. Therefore, there is a need to find an effective way to reduce stress, death anxiety, despair, and mental problems of the elderly. Logotherapy is focused on empowering patients to overcome the sad triangle of life, i.e. pain, sin, and suffering. Thus, the purpose of this study

\section{* Corresponding Author:}

Vahid Manzari Tavakoli, PhD

Address: Department of Educational Sciences, Farhangian University of Kerman, Kerman, Iran.

Tel: +98 (912) 4976329

E-mail: manzari.v63@gmail.com 
was to investigate the effectiveness of group logotherapy on death anxiety and life expectancy in the elderly people residing in the Kerman nursing center.

\section{Methods \& Materials}

The statistical population of this research includes all elderly residents of the Kerman nursing centers. Two centers of Amiralmo'menin and Mehrazin were randomly selected from the list of centers available at the Kerman Welfare Office. From these centers, a total of 30 people (18 women and 12 men) out of the total population of 37 people were selected using the convenient sampling method. These participants met the inclusion criteria for the study, i.e. age of 65 years or more, having a score below 96 in Miller's Life Expectancy Scale and a score more than 8 in Templar Death Anxiety Scale, and completed the consent form to participate in the research. The participants when then randomly divided into experimental and control groups (15 subjects in each group).

The sample size was determined to be at least 10 subjects in each group based on experimental research type, as mentioned previously [20]. It should be noted that homogeneity was maintained in both the groups with respect to age, gender, physical ability and the level of education. In the experimental group, group logotherapy was taught in 10 sessions (90 minutes per session). During this period, the control group did not receive any intervention. Both the groups were required to answer Miller's Life Inventory Scale and Templar Death Anxiety Scale before and after the intervention. Data were analyzed using statistical methods such as frequency, percentage, mean, standard deviation, and analysis of multivariate covariance analysis.

\section{Results}

Based on demographic data, most of the subjects were in the age group of 70 to 72 years $(34.88 \%)$. In this study, about $81 \%$ of the elderly were married. Most of the elderly participants had a diploma degree (41.75\%). It should be noted that the demographic variables of the sample group and the variables of death anxiety and life expectancy were compared before intervention using the Mann-Whitney test and t test. Results showed that there was no significant difference in experimental and control groups in terms of demographic variables, death anxiety, and life expectancy (Table 1). As seen in Table 1, the mean scores and standard deviation of the experimental group in post test has changed dramatically compared to the pre-test.

To check the precondition of homogeneity of variance, the Levine test was used which showed the amount of 0.412 and a significant level of 0.21 whose insignificance indicated the homogeneity of variances. To verify the normal distribution of data, the Kolmogorov-Smirnov test was used. Its results were not significant, which indicates that the distribution of the pre-test variables is normal. Results of analysis of covariance of experimental and control groups on death anxiety showed that there is a significant difference in death anxiety between both the groups with pre-test control $(\mathrm{F}=20.21$ and $\mathrm{P}<0.01)$. Thus, this indicates that group logotherapy reduces death anxiety in the elderly of the experimental group. Analysis of covariance of experimental and control groups in the life expectancy variable showed that there is a significant difference in terms of life expectancy between both the groups with pre-test control $(\mathrm{F}=23.98$ and $\mathrm{P}<0.01)$. This indicates that group logotherapy increased life expectancy in the elderly of the experimental group.

\section{Conclusion}

In general, it can be concluded that group logotherapy can reduce death anxiety and increase life expectancy of the elderly residents of nursing centers and subsequently can increase their mental health. Therefore, having meaning in life, especially in elderly residents of nursing cen-

Table 1. Mean and standard deviation of life expectancy and death anxiety in experimental and control groups before and after intervention

\begin{tabular}{ccccccc}
\hline \multirow{2}{*}{ Group } & Test & \multicolumn{2}{c}{ Pre-Test } & \multicolumn{2}{c}{ Post-Test } \\
\cline { 3 - 6 } & & Index & Death Anxiety & Life expectancy & Death Anxiety & Life expectancy \\
\hline \multirow{2}{*}{ Control } & Mean & 9.88 & 37.14 & 9.47 & 17.52 \\
& Standard Deviation & 2.32 & 8.63 & 2.45 & 7.19 \\
\multirow{2}{*}{ Experiment } & Mean & 9.65 & 27.56 & 6.12 & 69.97 \\
& Standard Deviation & 2.13 & 8.72 & 2.41 & 8.58 \\
\hline
\end{tabular}


ters, has an important and undeniable role in reducing the death anxiety, boosting life expectancy, and effective coping with events of life.

\section{Acknowledgments}

This research did not receive any specific grant from funding agencies in the public, commercial, or not-forprofit sectors.

\section{Conflict of Interest}

All authors certify that this manuscript has neither been published in whole nor in part nor being considered for publication elsewhere. The authors have no conflicts of interest to declare. 


\title{
اثربخشى معنادرمانى كَروهى بر اضطراب مركَى و اميد به زندكَى سالمندان مقيهم مراكز شبانهروزى شهر كرمان
}

اميرحسين حاجى عزيزى'، بهناز بهمنى"، نفيسه مهدى"! "وحيد منظرى توكلى"، اديبه برشانه

1- كروه روانشناسى مشاوره، دانشكده علوم انسائى، دانشعاه اساميشن، بانكوك، ثايلند.

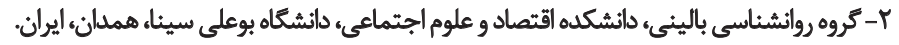

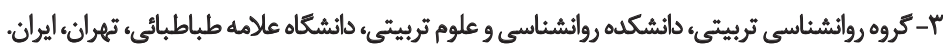

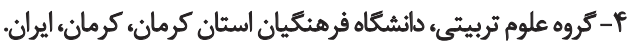

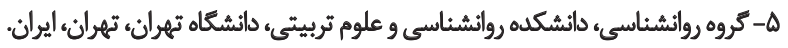

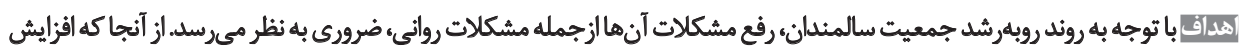

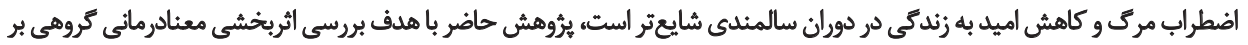

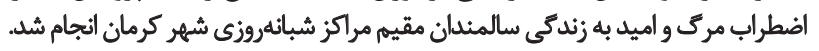

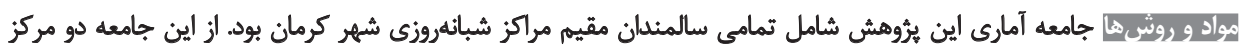

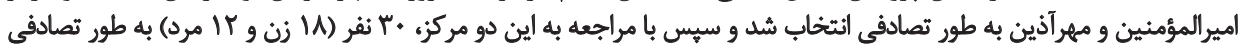

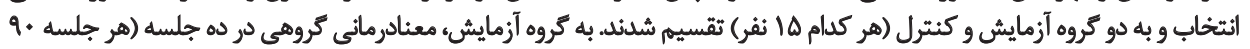

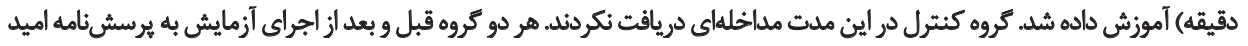

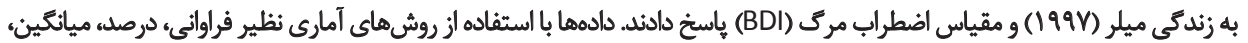

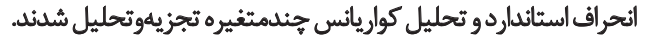

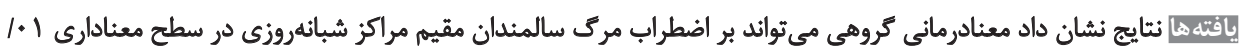

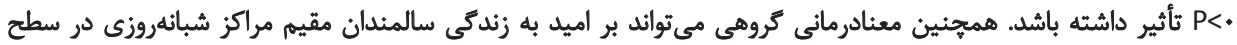

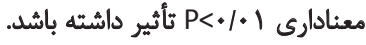

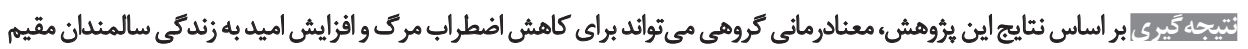

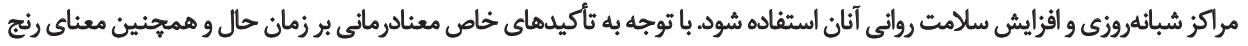

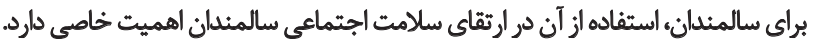

$$
\text { كمليدواروها: }
$$

مى ييوندد. تغييرات ذكرشده در مسائل خاص اين دوره تحولى به

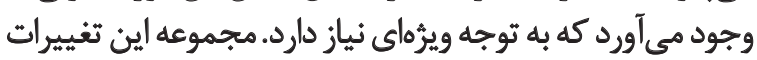

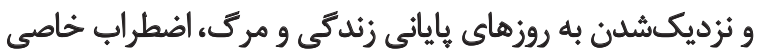

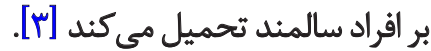
در ميان انواع خاصى از اضطراب كه بر اساس منشأ آن شناخته

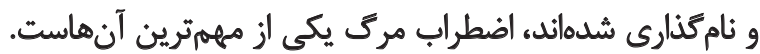

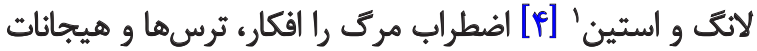

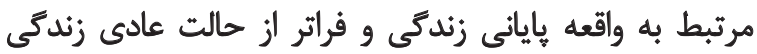

1. Lang and Stein

مقدمه

برآوردها حاكى از آن است كه ثا سال •r.r.r جمعيت

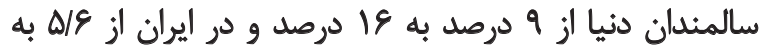

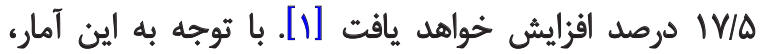

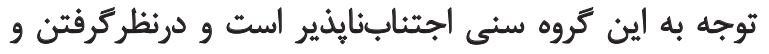

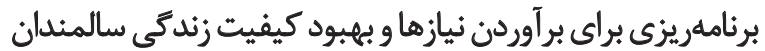

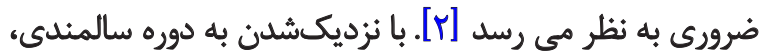

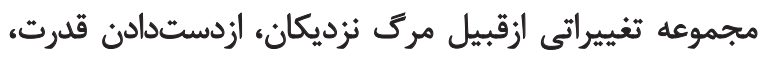

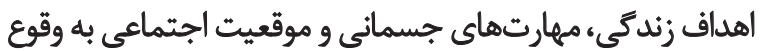

ㄷ.?.
\end{abstract}

: نويسئده مسئول:

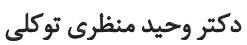

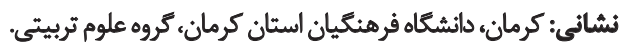

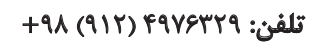
manzari.v63@gmail.com بطئ الكترونيكي 
است [ [ [] انسان در سطح وجودى مثفاوتى زندكى مي كند، مثل

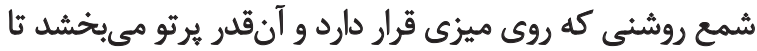

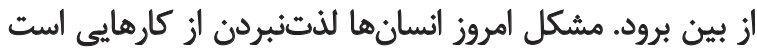

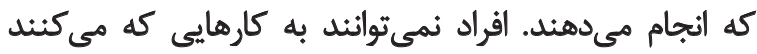

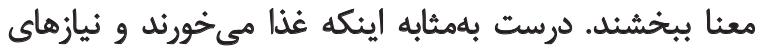

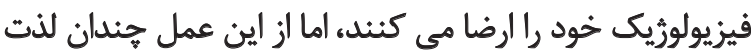

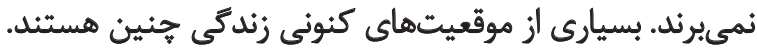

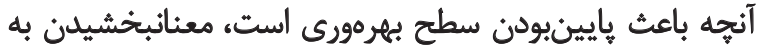

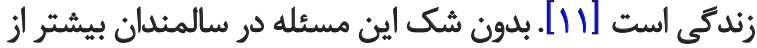
ساير دورههاى رشد انسان مشاهده مي شيود.

تلاش براى يافتن معنا در زندگى انسان اساسىترين نيروى ابرى

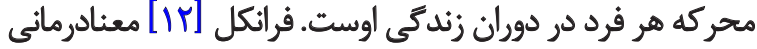

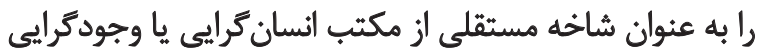

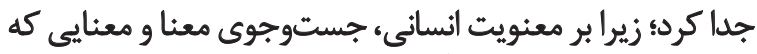

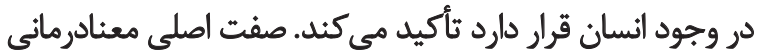

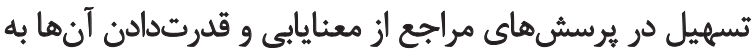

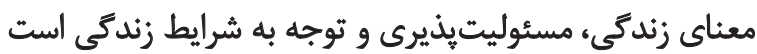

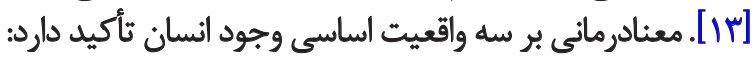

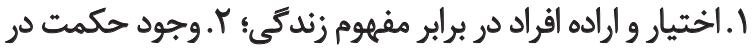

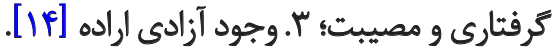

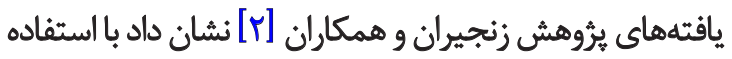

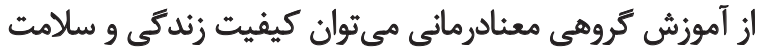

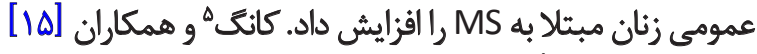

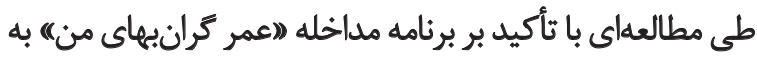

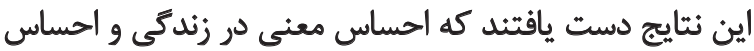

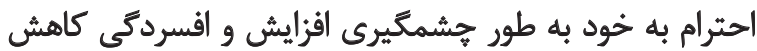

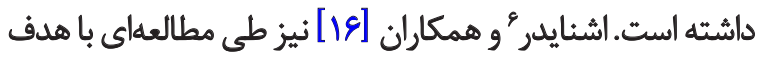

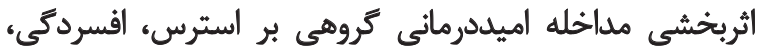

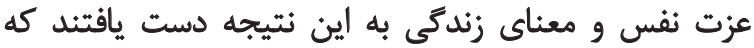

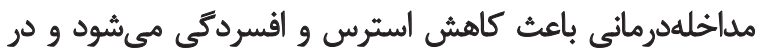

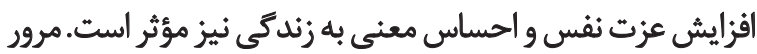

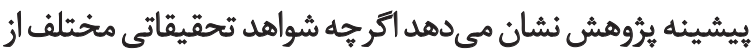

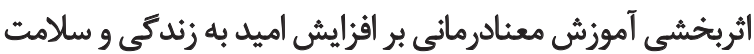

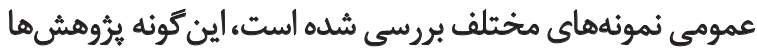

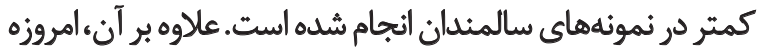

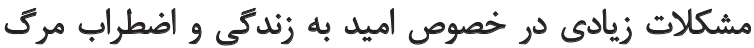

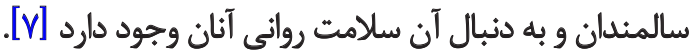

حفظ سلامت روان سالمندان در دنياى مدرن امروزى يكى از

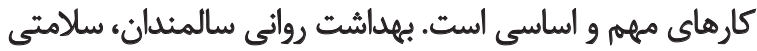
روانى آنها را به دنبال دارد و شاخصهايى از شادئيست رواني

5. Kang

6. Snyder

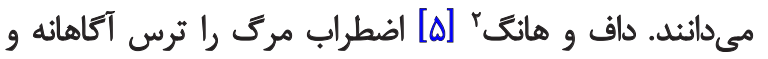

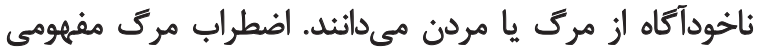

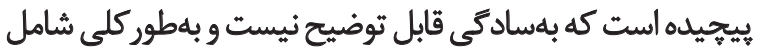

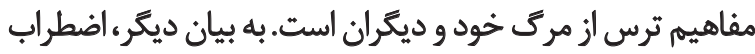

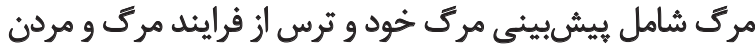

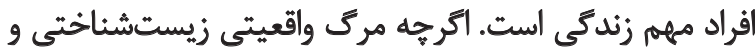

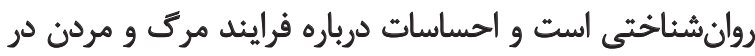

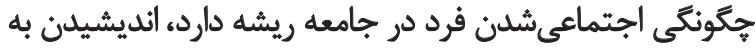

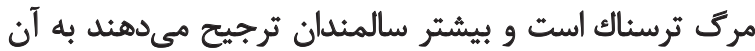

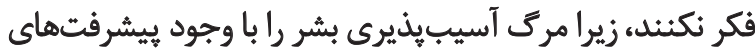

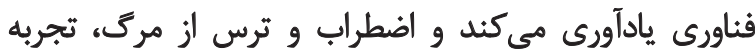

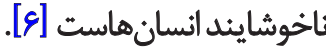

اميد به زندكى نيز شاخصى آمارى است كه نشان مي دهد متوسط

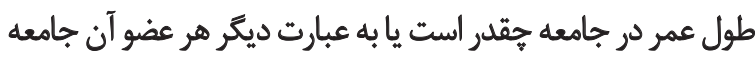

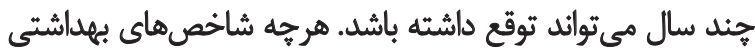

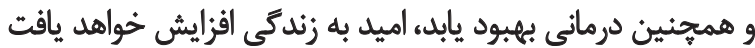

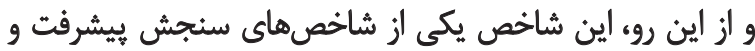

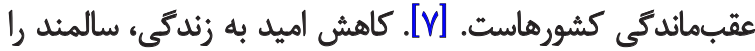

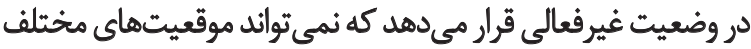

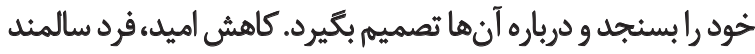

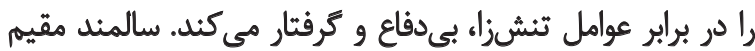

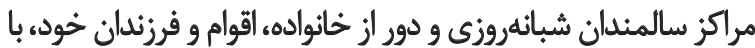

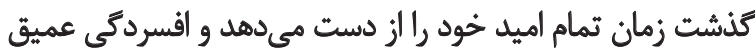

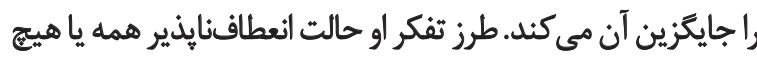

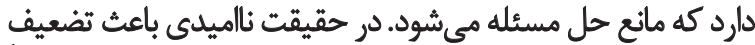

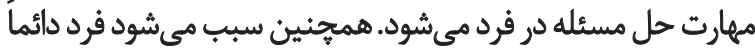

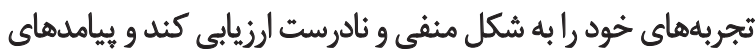

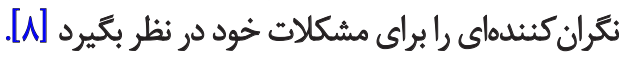
با توجه به اين مطلب كه حالات روانشناختى و فشارهاي

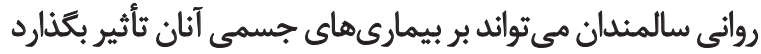

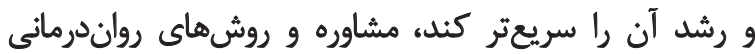

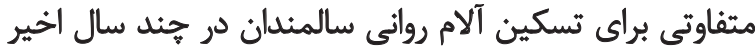

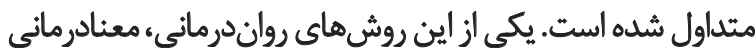

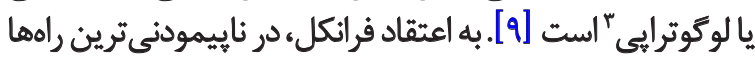

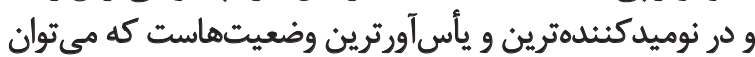

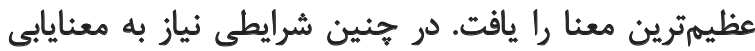

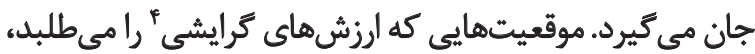

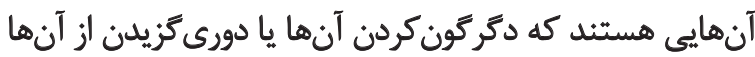

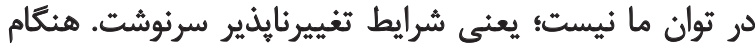

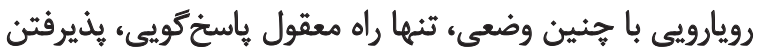

4. Attitudinal values 
كرمان فهرست مراكز سالمندان تهيه شد و دو مركز خصوصى

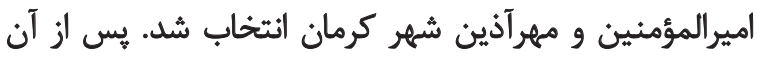

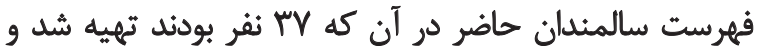

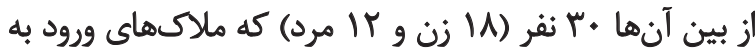

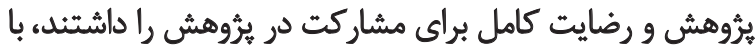

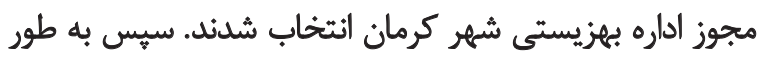

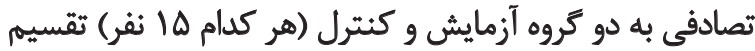

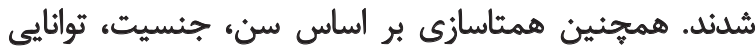

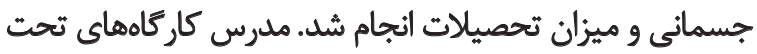

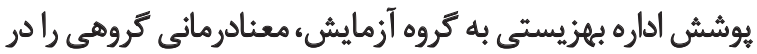

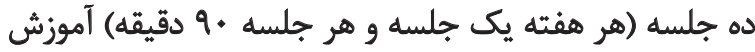
داد. گروه كنترل در اين مدت مداخلهاى دريافت نكرد.

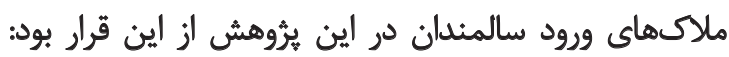

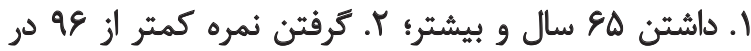

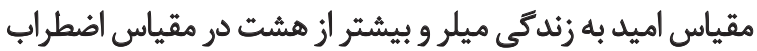

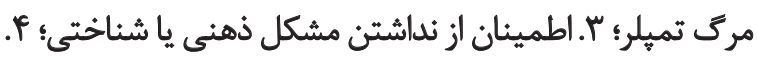

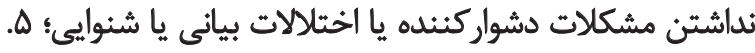

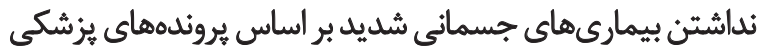
آنان؛ 8. تمايل براى شركت در بروهش.

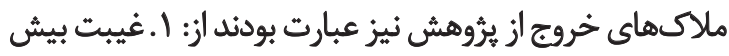

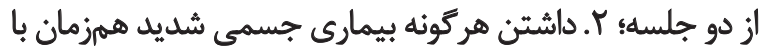

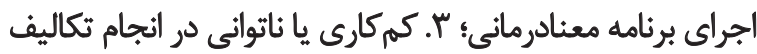

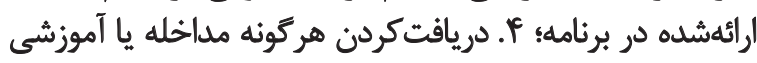

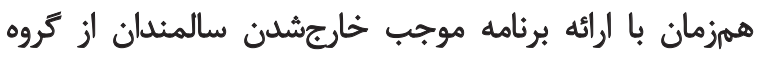

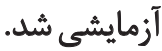

ملاحظات اخلافى اين مطالعه اين موضوعات را دربر داشت:

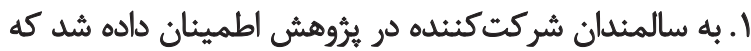

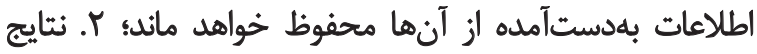

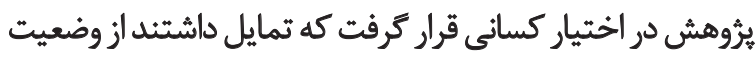

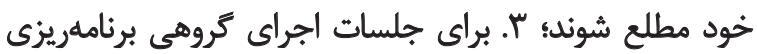

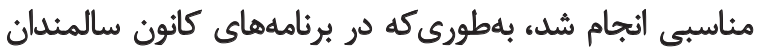

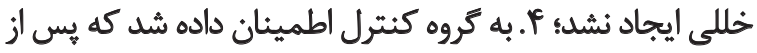

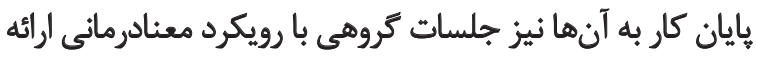

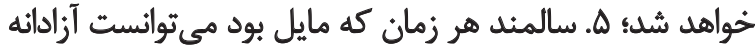

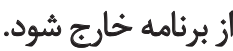

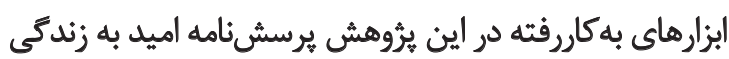

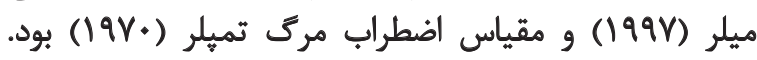

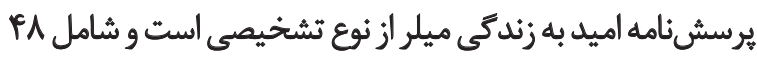

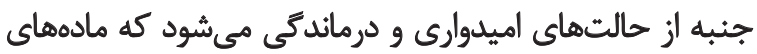

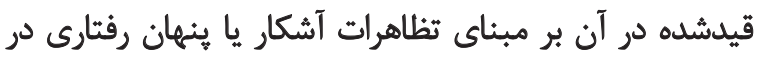

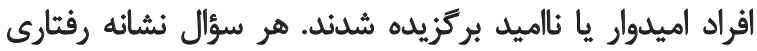

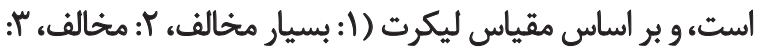

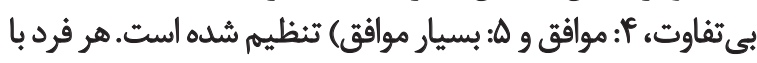

اميدوارى سالمند را ايجاد مى كند كه براى فرد سالمند، خانواده

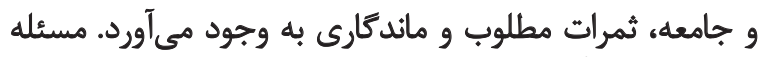

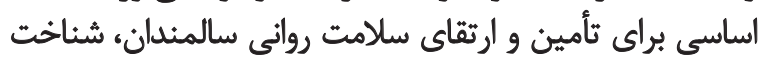

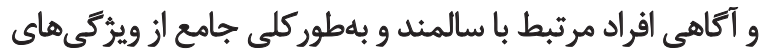

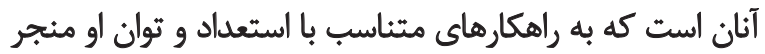

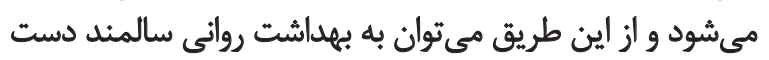

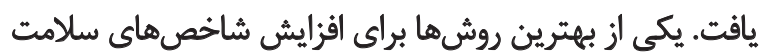

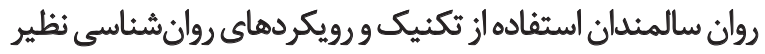

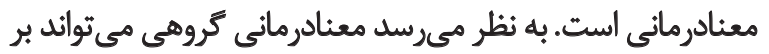

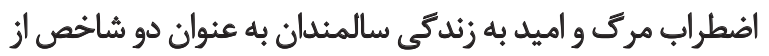
شاخصهاى مهم سلامت روان تأثيرى عميق داشته باشند. تجربه تروهى فرصتى ارزشمند براى برقرارى رابطهاى واقعى دري

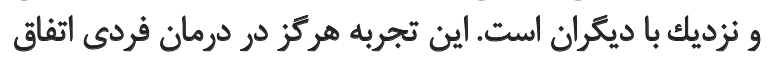

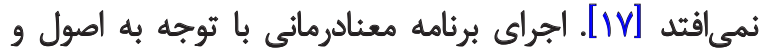

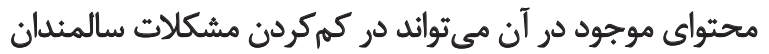

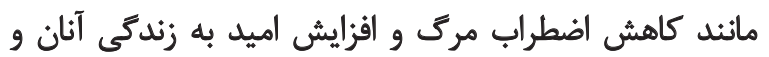

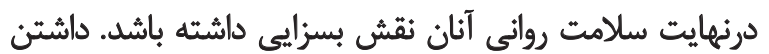

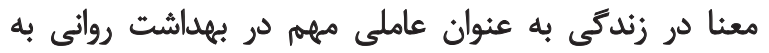

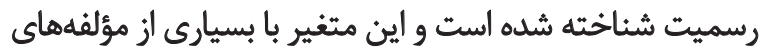

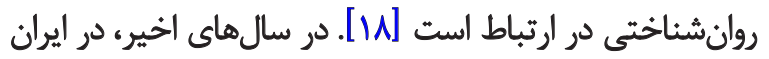

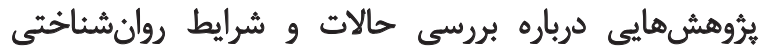

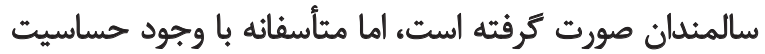

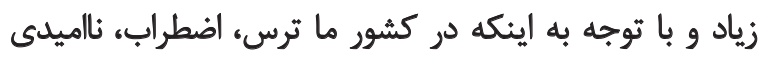

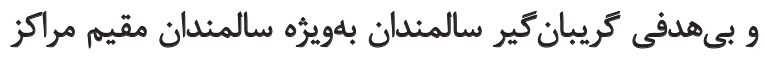

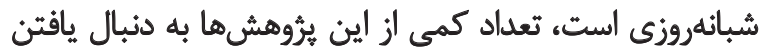

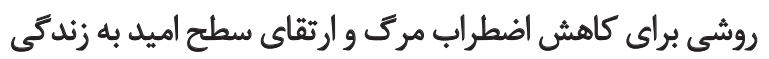

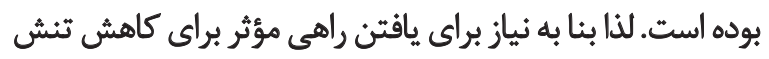

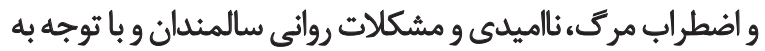

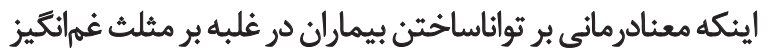

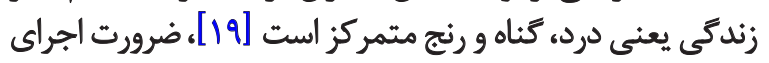

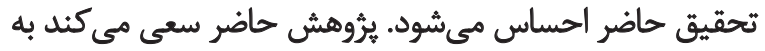

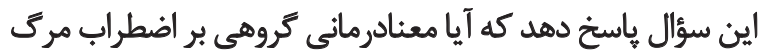

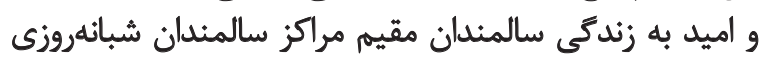
شهر كرمان تأثير معنادارى دارد يا خير. روثُ مطالعه اين بثروهش از نوع كاربردى با روش تجربى، با كروه كنترل

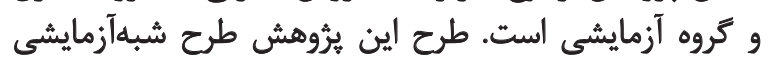

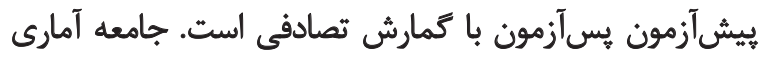

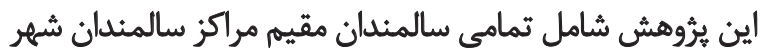

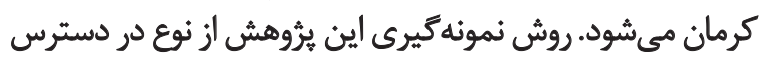

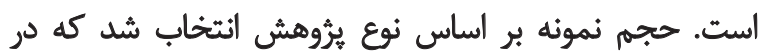

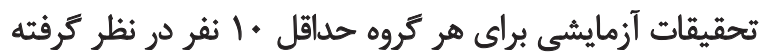

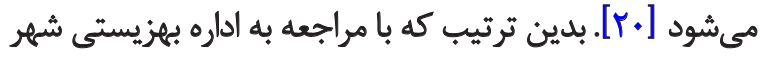


شرايط قرار ندارد و اين خود اوست كه حق انتخاب دارد كه با شرايط مقابله كند.

جلسات سوم، جهارم و گينجم: مرور تكاليف جلسه قبل،

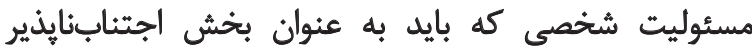

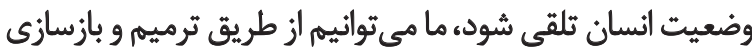

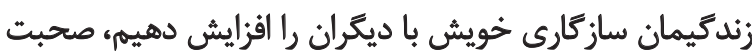

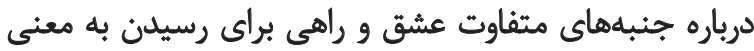

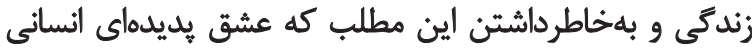

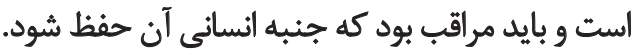

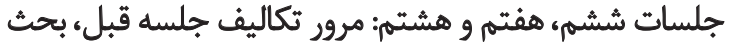

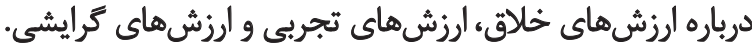

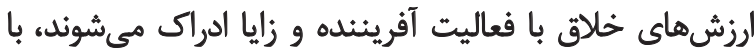

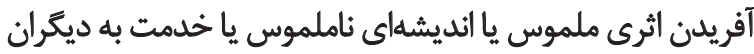

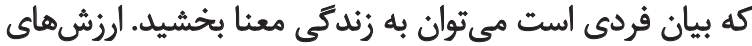

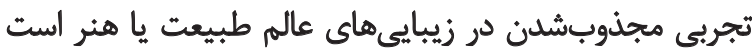

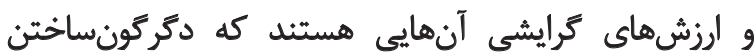

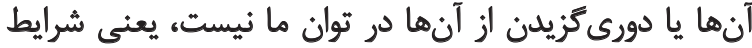

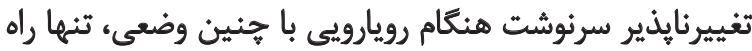

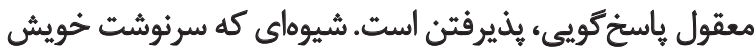

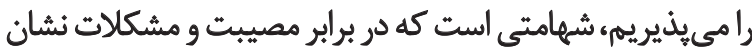

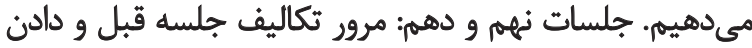

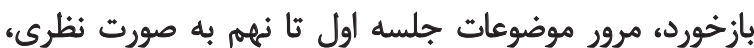
اجمالى و عملى، اجراى يس آزمون.

يافتهها

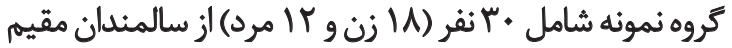

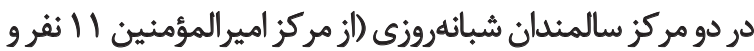

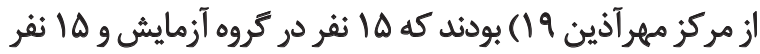

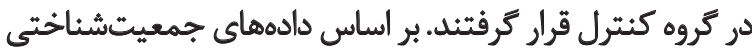

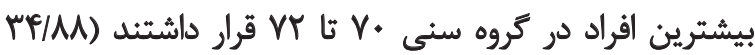

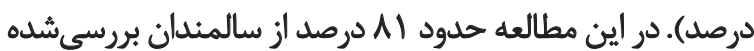

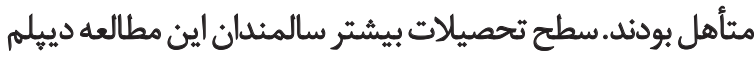

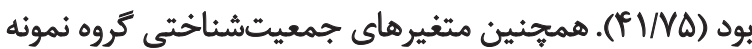

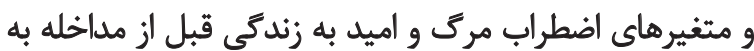

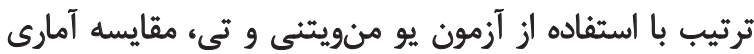

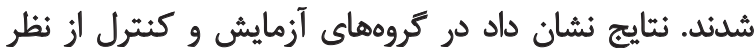

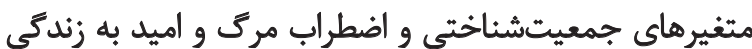

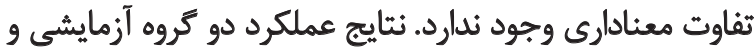

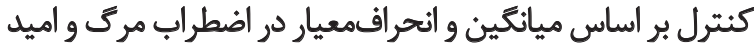

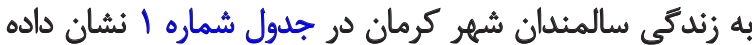

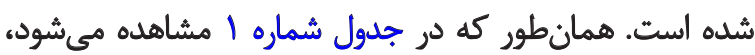

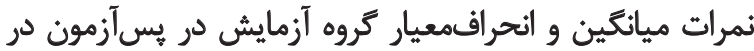
مقايسه با بيشآزمون تغيير جشمغيرى كرده است
انتخاب جملهاي كه درباره او صدق مى كند، امتياز كسب مى كنيد.

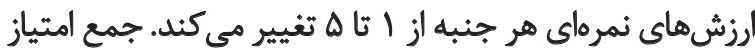

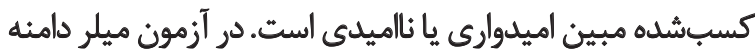

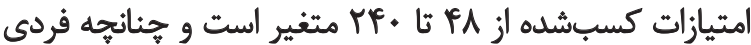

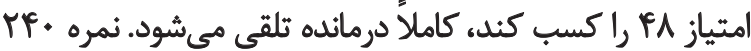

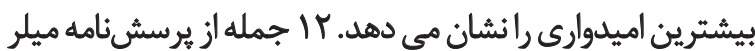

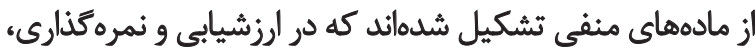

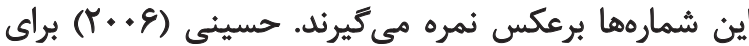

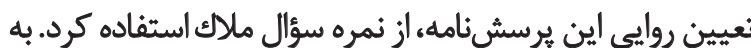

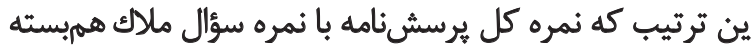

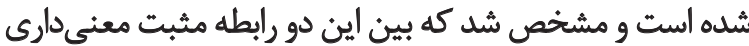

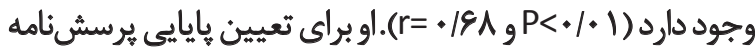

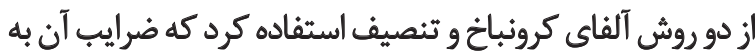

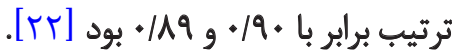

تميلر در سال •19V مقياس اضطراب مرك را ساخت كه

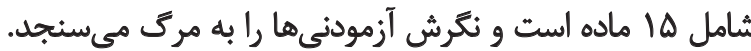

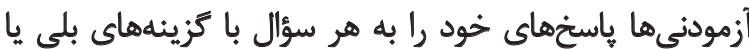

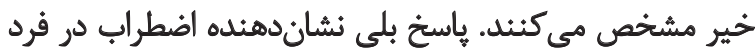

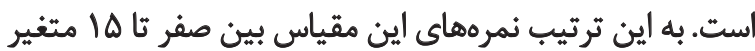

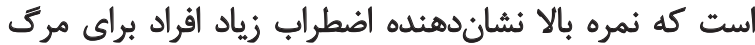

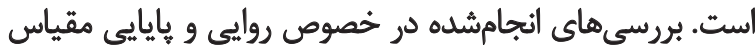

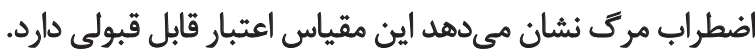

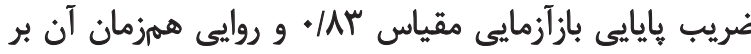

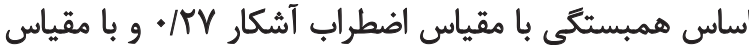

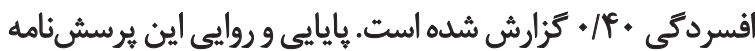

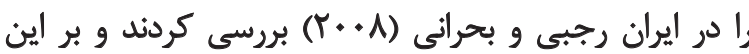

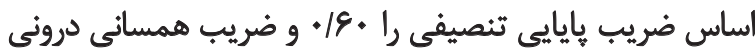
را

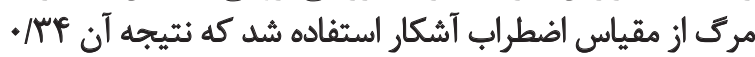

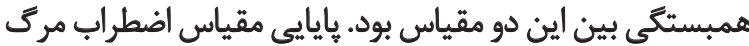

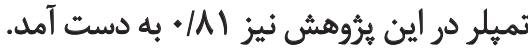
براى تجزيهوتحليل دادهها از روشهاى آمار توصيفى نظير براني

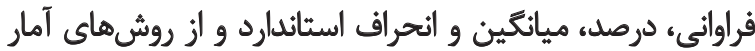

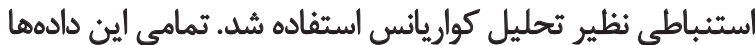

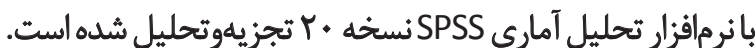

$$
\text { روش مداهله }
$$

طرح كلى جلسات معنادرمانى كروهى به اين صورت بودي:

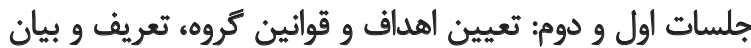

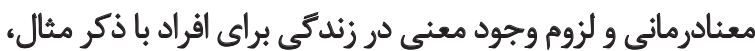

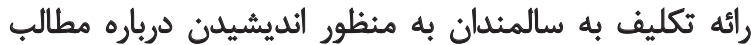

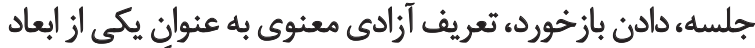
هستى انسان و بيان اين موضوع كه انسان كاملاً تحت سلطه بله إنه 
جدول ا. ميانكين و انحرافمعيار نمره اميد به زندكى و اضطراب مرك كروههاي آزمون وكنترل، قبل و پيس از مداخله

\begin{tabular}{|c|c|c|c|c|c|}
\hline \multicolumn{2}{|c|}{ يس أزمون } & \multicolumn{2}{|c|}{ ييثىأزمون } & \multicolumn{2}{|c|}{ أزمون } \\
\hline اميد به زندكى & اضطراب مركى & اميد به زندكى & اضطراب مرك & شاخص & تروه \\
\hline $\mathrm{n} / \mathrm{Ta}$ & $9 / M^{e}$ & $r / f i$ & $\checkmark / M$ & مياثكين & \\
\hline V/9I & r/Af & NHE & T/M & أتحر|ف استثائدارد & كتترل \\
\hline$q \& / v q$ & $g / \pi$ & $V / \& \Delta$ & $9 / \Delta F$ & مياكين & \\
\hline NAS & $r / N F$ & NTY & T/M & انحراف استثاندارد & ارمايس \\
\hline
\end{tabular}

닌

جدول r. نتايج آزمون كولموكروف اسميرنف در متغيرهاي اضطراب مرك و اميد به زندكي

\begin{tabular}{|c|c|c|c|}
\hline 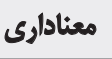 & آزمون كولموكروف السميرنف & متغيرها & \\
\hline.$/ T V D$ &.$/ A \mathrm{~W}$ & اضطراب مرى & ييش آزهون \\
\hline . &.$/ \& \Delta \Delta$ & اميد به زندكى & ييش أزمون \\
\hline
\end{tabular}

DU

آزمودنى هاى كروه آزمايش و كواه از لحاظ اضطراب مركى تثاوت

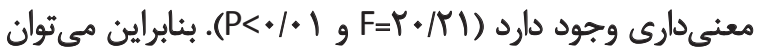

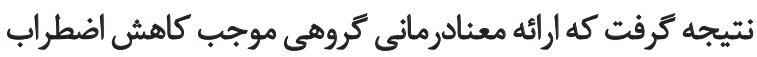

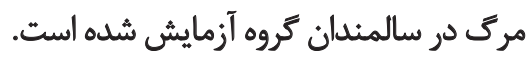

نتايج تحليل كوواريانس كروههاى آزمايشى و كنترل در متغير

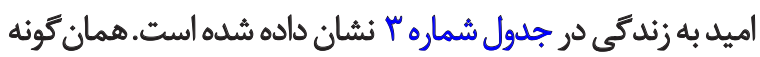

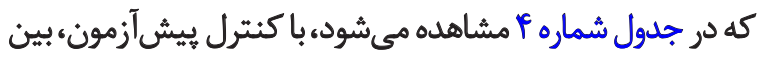

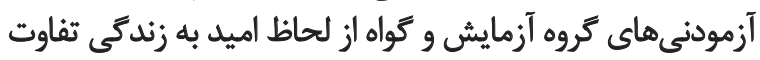

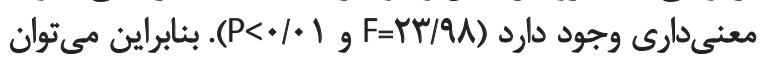

در اين يُروهش براى بررسى بيشفرض همكنى واريانس از

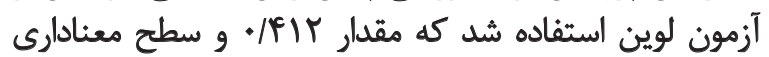

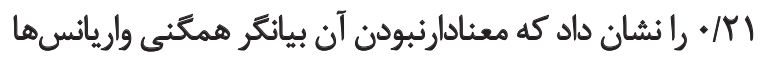

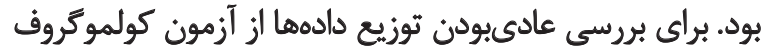

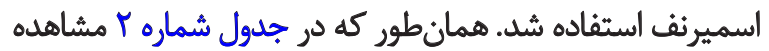

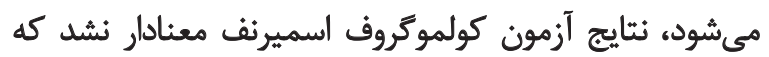

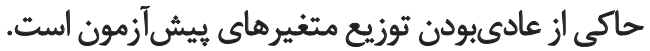
نتايج تحليل كواريانس كروههاى آزمايشى و كنترل در متغير

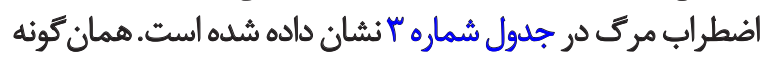

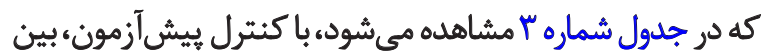

جدول با. نتايج تحليل كوواريانس مقايسه نمرههاى يسآزمون كروه آزمايش با كروه كثترل در اضطراب مرى

\begin{tabular}{|c|c|c|c|c|c|}
\hline سطح معنادارى & تسببت F & ميانكين مجذورها & ورجه أزادى & مجموع مجذورات & منابع تغييرات \\
\hline.$/ 1 F$ & $1 / M^{\infty}$ & PV/VA & 1 & PV/VA & ييش آزمون \\
\hline \multirow[t]{2}{*}{.1 .1} & $r M / r$. & $\Delta A T / F T$ & 1 & $\Delta A Y / T H$ & كروها \\
\hline & & $\mathrm{rV} / \mathrm{FV}$ & rV & $n+1 / n$ & خطا \\
\hline
\end{tabular}

L

جدول F. نتايج تحليل كوواريانس مقايسه نمرههاي يسآزمون كروه آزمايش با كروه كنترل در اميد به زندكى

\begin{tabular}{|c|c|c|c|c|c|}
\hline سطح معنادارى & Fسبت F & مياتكَين مجذورها & درجه أزادى & مجموع مجذورات & منابع تغييرات \\
\hline.$/ 1 r$ & $\mid / M$ & $\Delta 1 / 91$ & 1 & (1)/9F & ييش آزمون \\
\hline \multirow[t]{2}{*}{.1 .1} & $M / Q$ & IXV/tee & 1 & IIAV/Fe & $\log 5$ \\
\hline & & PQ/DI & TV & 1.8810 & خطا \\
\hline
\end{tabular}

il 
همجنين در مداخله كروهي معنادرمانى اصل ئيرش مسئوليت

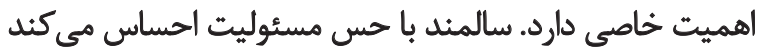

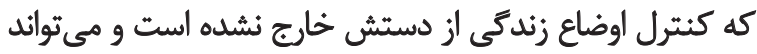

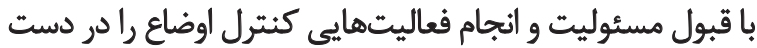

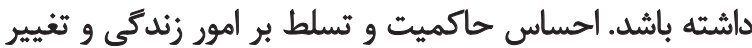

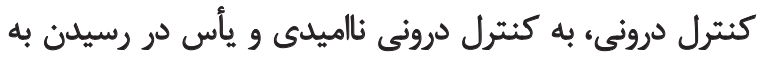

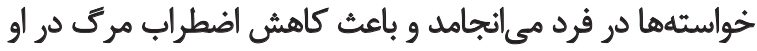

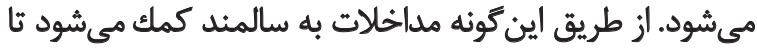

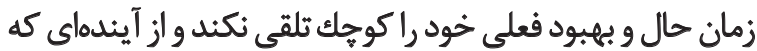
هنوز نيامده است، ترس نداشته باشد.

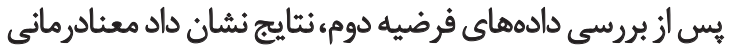

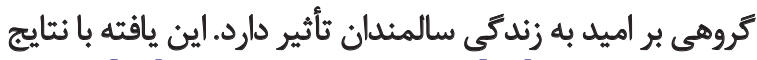

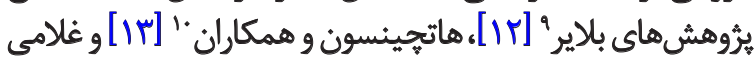

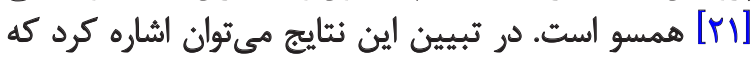

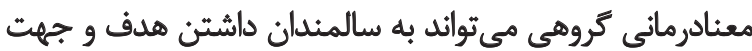

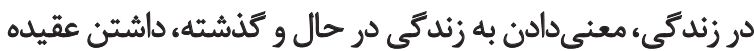

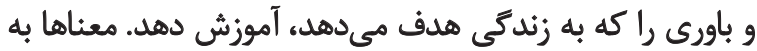

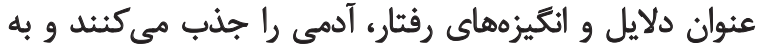

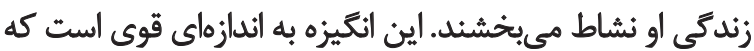

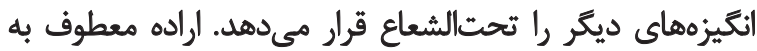

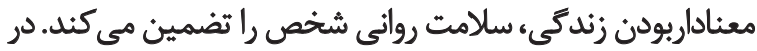

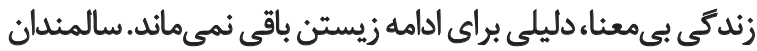

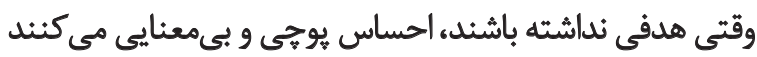
و در خلئى بدون تريز ترفتار مى آيند.

درحقيقت، اين معناست كه به انسان جرئت بودن و شدان

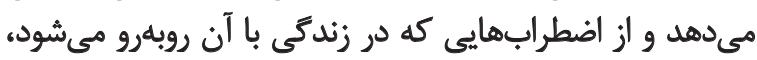

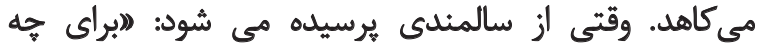

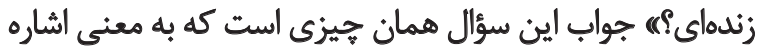

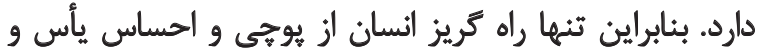

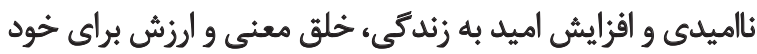

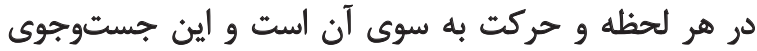

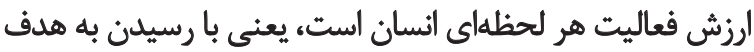

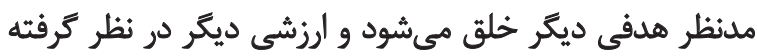

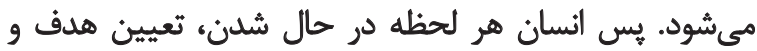

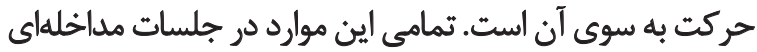

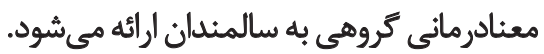

از طرف ديكر، هر گروهى به تناسب وضع خود از نظر درمانكر

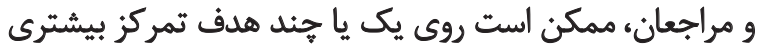

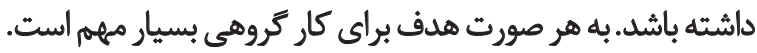

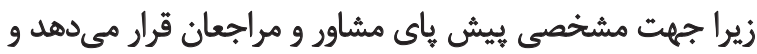
براى درمائكر اين امكان را فراهم ميى كند كه در خاتمه كار بات باتوجه

9. Blair

10. Hutchinson et al.
نتيجه گرفت كه ارائه معنادرمانى گروهى موجب افزايش اميد به زندكى در سالمندان كروه آزمايش شده است

ث:

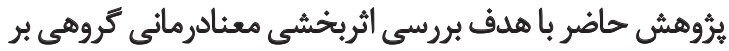

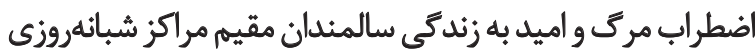

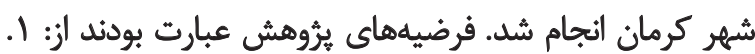

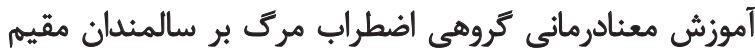

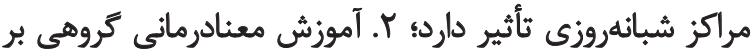
اميد به زندكى سالمندان مقيم مراكز شبانهروزي تأثير دأثير داردي.

يس از اجراى مداخله و بررسى دادههاى فرضيه اول نتايج نشان

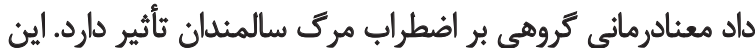

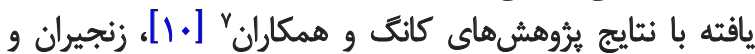

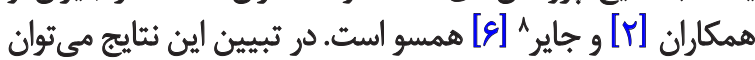

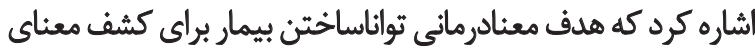

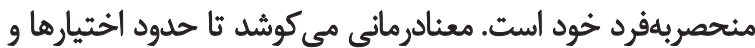

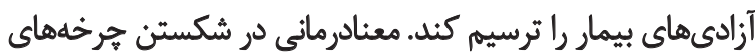

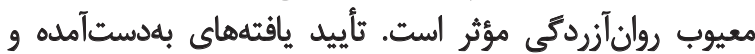

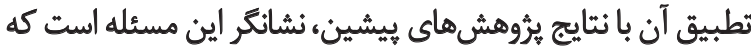

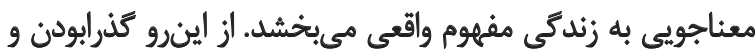

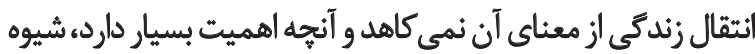

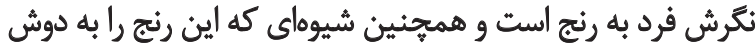

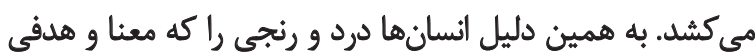

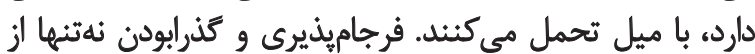

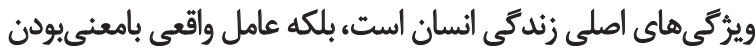

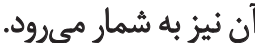

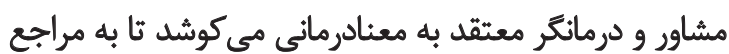

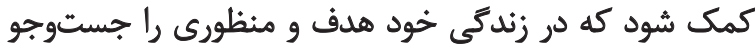

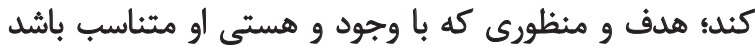

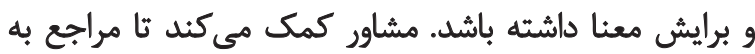

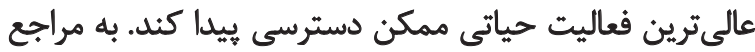

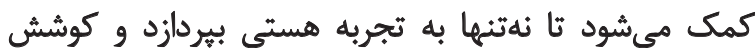

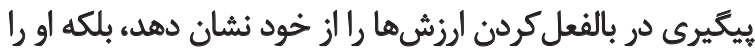

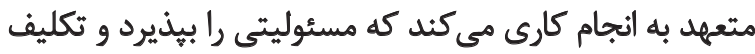

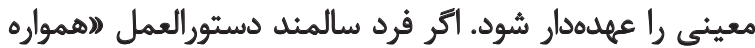

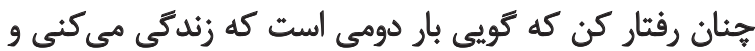

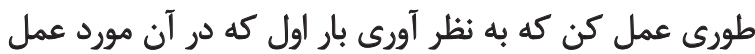

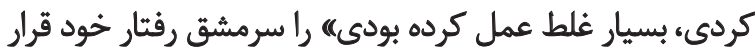

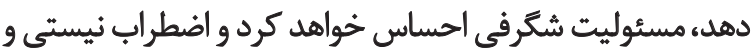
فانىشدن كاهش خواهد يافت.

7. Kang et al

8. Gire 


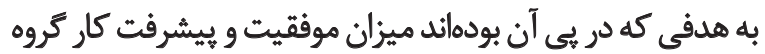

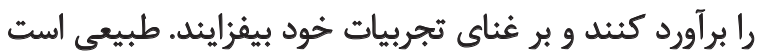

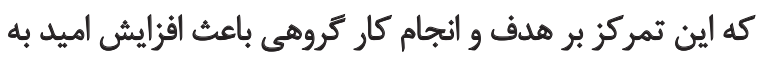

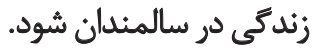

از آنجا كه معنادرمانى فلسفهاى از زندكى را آموزش مي دهد كه

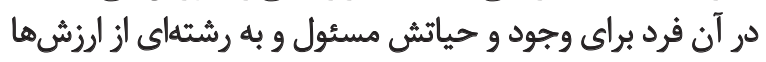

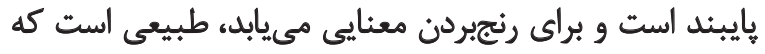

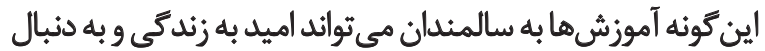

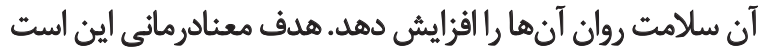

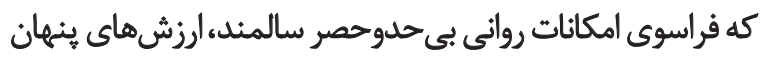
و بدون دستكارى رسيدن به واقعيت را به او آموزش دها لهد.

\section{تتيجهَيرى نهايى}

در هايان مى توان نتيجه ترفت مداخلات و شيوههاى معنادرمانى

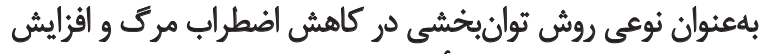

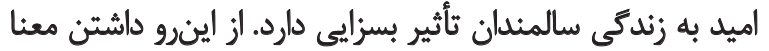

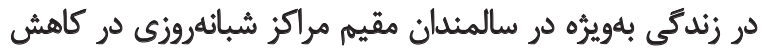

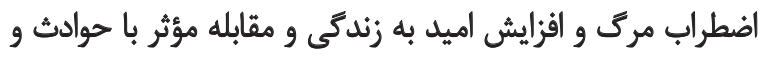

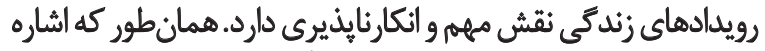

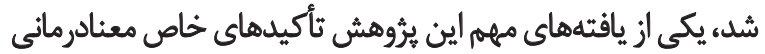

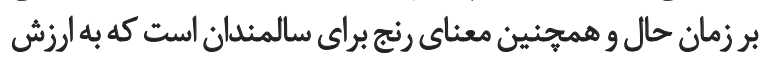
كاربردى آن در ارتقاى سلامت اجتماعى سالمندان اشاره مى كنئ.

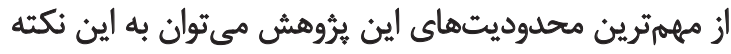

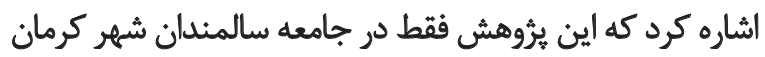

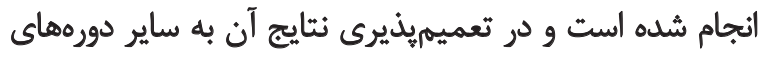

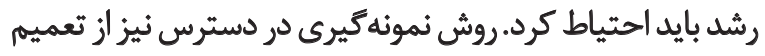

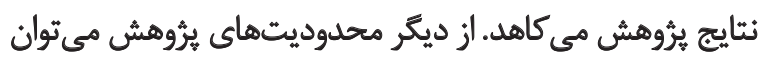

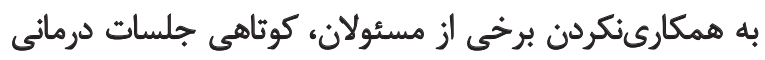

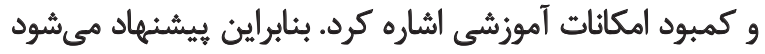

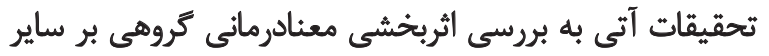

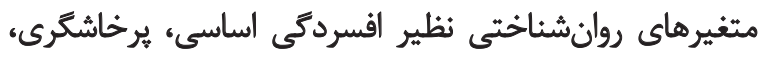

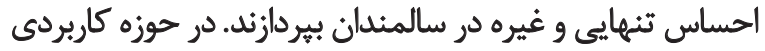

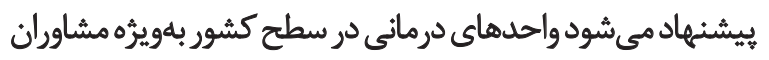

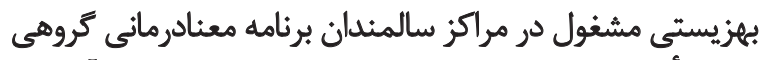

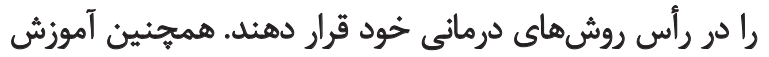

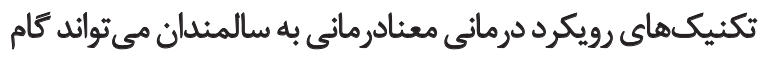
مؤثرى در بهبود سلامت روانى و جسمانى آنان باشد.

$$
\text { تشكر و قدردانى }
$$

از تمامى مسئولان و كاركنان محترم دو مركز خصوصى

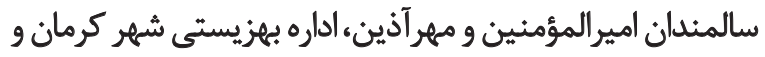

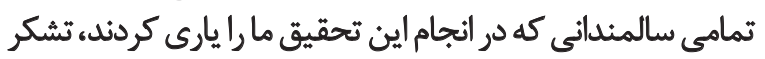
و قدردانى مى كنيم. مقاله حامى مالى نداشته استه است. 


\section{References}

[1] Rastabi A, Sharifi AA, Refahi J. [Psychometric properties of geriatric depression scale in an Iranian sample (Persian)]. Iranian Journal of Ageing. 2013; 8(2):54-9.

[2] Zanjiran S, Borjali A, Kraskian A. [Effect of group logotherapy education on quality of life and general health in women of sanatorium (Persian)]. Razi Journal of Medical Sciences. 2015; 21(127):39-51.

[3] Sheibani TF, Pakdaman S, Hassanzadeh TM. [The effect of reminiscence on depression and loneliness in elderly (Persian)]. Applied Psychology. 2010; 4(1):26-39.

[4] Lang AJ, Stein MB. Anxiety disorders. How to recognize and treat the medical symptoms of emotional illness. Geriatrics. 2001; 56(5):24-7. PMID: 11373949

[5] Duff RW, Hong LK. Age density, religiosity and death anxiety in retirement communities. Review of Religious Research. 1995; 37(1):19. doi: $10.2307 / 3512068$

[6] Gire J. How death imitates life: Cultural influences on conceptions of death and dying. Online Readings in Psychology and Culture. 2014; 6(2). doi: 10.9707/2307-0919.1120

[7] Ghara Zibaei F, Aliakbari Dehkordi M, Alipour A, Mohtashami T. [Efficacy of group logo therapy in the perceived stress and life expectancy in MS patients (Persian)]. RPH. 2013; 6(4):12-20.

[8] Nasiri Z, Dadkhah A, Khodabakhshi Koulai A. [Investigate and comparsion self-esteem and happiness among residential and non-residential old people (Persian)]. Iranian Journal of Ageing. 2012; 7(2):18-25.

[9] Yazdanbakhsh N, Younesi SJ, Aazimiyan S, Foroughan M. [Effects of group counseling based on logo therapy to decrease loneliness in elderly men (Persian)]. Iranian Journal of Ageing. 2016; 11(3):392-9.

[10] Kang KA, Im JI, Kim HS, Kim SJ, Song MK, Sim S. The effect of logotherapy on the suffering, finding meaning, and spiritual well-being of adolescents with terminal cancer. Journal of Korean Academy of Child Health Nursing. 2009; 15(2):136-44. doi: 10.4094/jkachn.2009.15.2.136

[11] Ejei J, Manzari Tavakoli V, Hosseini SR, Hashemizadeh V. [Analysis of the effectiveness of group cognitive behavioral therapy, group logo therapy and their combination in the increase of social adjustment in maladjustment students (Persian)]. Journal of Psychological Health. 2012; 6(3):30-9.

[12] Blair RG. Helping older adolescents search for meaning in depression. Journal of Mental Health Counseling. 2004; 26(4):33347. doi: 10.17744/mehc.26.4.w8u9h6uf5ybhapyl

[13] Hutchinson GT, Chapman BP. Logo therapy enhanced REBT: An integration of discovery and reason. Journal of Contemporary Psychotherapy. 2005; 35(2):145-55. doi: 10.1007/s10879-005$2696-x$

[14] Ebrahimi A. [Comparison the effectiveness of psychotherapy integration of religious orientation, cognitivebehavioral therapy and medication on depression and attitudes of patients with dysthymic disorder (Persian)] [MSc. dissertation]. Isfahan: Isfahan University; 2017.

[15] Kang KA, Im JI, Kim HS, Kim SJ, Song MK, Sim S. The effect of logo therapy on the suffering, finding meaning, and spiritual well-being of adolescents with terminal cancer. Journal of Korean
Academy of Child Health Nursing. 2009; 15(2):136. doi: 10.4094/ jkachn.2009.15.2.136

[16] Snyder CR, Ilardi SS, Cheavens J, Michael ST, Yamhure L, Sympson S. Cognitive Therapy and Research. 2000; 24(6):747-62. doi: 10.1023/a:1005547730153

[17] Zhou W, He G, Gao J, Yuan Q, Feng H, Zhang CK. The effects of group reminiscence therapy on depression, self-esteem, and affect balance of Chinese community-dwelling elderly. Archives of Gerontology and Geriatrics. 2012; 54(3):440-7. doi: 10.1016/j. archger.2011.12.003

[18] Brody CM, Semel VG. Strategies for therapy with the elderly: Living with hope and meaning. New York: Springer Publishing Company; 2005

[19] Colman PG, O'Hanlon A. Ageing and development : Theories and research. New York: Oxford University Press; 2013.

[20] Hooman HA. [Understanding the scientific method in the behavioral sciences (Persian)]. Tehran: SAMT; 2006.

[21] Gholami M, Pasha GH, Sodani M. [To investigate the effectiveness of group logo therapy on the increasement of life expectancy and health on female teenager major thalassemia patients of Ahvaz city (Persian)]. Knowledge \& Research in Applied Psychology. 2010; 11(42):23-42.

[22] Hosseini, SM. [Relationship of life expectancy and psychological hardiness in male and female students (Persian)] [MSc. thesis] Gachsaran: Islamic Azad University of Gachsaran Branch; 2006. 
